\title{
A urine analysis method suitable for children's nappies
}

Adrian Edwards, Judith van der Voort, Robert Newcombe, Hazel Thayer, Kate Verrier Jones

\begin{abstract}
Background-Urinary tract infection in infancy continues to be underdiagnosed, despite its association with renal scarring and thus hypertension, renal failure, and other sequelae. Low ascertainment of urinary tract infections reflects the many difficulties in establishing a diagnosis, some of which could be eliminated by a simple, reliable method for preliminary investigation of children's urine.

Aim-To assess the accuracy of a new, simple method for testing urine for nitrite and leucocyte esterase, which could be applied to children in primary care.

Methods-An in vitro study was carried out to compare the results of conventional urine analysis with urine analysis on urine soaked on to panty-liners, and with the laboratory investigation. Two urine analysis stick types were used (Boehringer Mannheim Nephur sticks and Bayer Multistix 8SG) and two brands of panty-liners. Analysis examined evidence of agreement and bias for different methods in addition to sensitivity, specificity, and negative predictive values for urine analysis.

Results-Pressing urine analysis test sticks on to panty-liners soaked with urine achieved consistent results compared with the results of conventional dipstick urine analysis. At a prevalence of $21.8 \%$, sensitivity and negative predictive values of urine analysis for laboratory confirmed urinary tract infection were $94 \%$ and $98 \%$, respectively, for Boehringer sticks, and $76 \%$ and $93 \%$, respectively, for Bayer sticks. At prevalences of $5 \%$ and $1 \%$ (prevalences that could be expected in primary care) Bayer sticks had negative predictive values of $98.7 \%$ and $99.7 \%$, respectively, and Boehringer sticks had values of $99.6 \%$ and $99.9 \%$, respectively.

Conclusions-Testing urine on pantyliners is accurate compared with conventional urine analysis. It may be possible to apply this method to testing unwell children presenting in primary care to identify those who require microbiological urine culture to confirm or eliminate a diagnosis of urinary tract infection. (F Clin Pathol 1997;50:569-572)
\end{abstract}

Keywords: children; urinary tract infection; urine analysis
Urinary tract infection (UTI) is a common illness in children, occurring in up to $5 \%$ of febrile presentations in primary care. ${ }^{1}$ Between $5 \%$ and $37 \%$ of children with a UTI have renal scarring when investigated in hospital, with the potential sequelae of hypertension, complications in pregnancy, and chronic renal failure. ${ }^{2}$ Risk factors associated with renal scarring include young age at first UTI, delay in starting treatment, and recurrent UTI. ${ }^{3-5}$ Early diagnosis and treatment is likely to prevent renal scarring, ${ }^{6}$ although the incidence and natural history of childhood UTI in primary care are not fully described.

There are several reasons why childhood UTI could go unrecognised in primary care, thus leaving a risk of renal scarring. The child may present with non-specific symptoms (especially fever) that are documented as other "routine" acute illnesses, without excluding UTI. If the diagnosis is considered, time pressures, difficulty in gaining parental cooperation, availability of laboratory services, and concerns about the cost of investigation all contribute to infrequent attempts to diagnose or exclude UTI. ${ }^{7}$ Often, when attempts are made, urine samples are collected in bags secured to the infant and transferred to a universal container for transport to the laboratory. Frequently, this method produces contaminated urine samples that are difficult to interpret.

All these difficulties may lower enthusiasm among general practitioners to pursue a diagnosis of UTI in young children. ${ }^{7}$ Many of the difficulties, however, would be overcome if a simple, reliable method distinguished children in whom a UTI could be confidently excluded, making formal urine investigation unnecessary, from those in whom a UTI was suspected. These latter children would require further microbiological investigation of urine to clarify the diagnosis or absence of UTI.

The relevant urine analysis stick tests for UTI are those for leucocyte esterase and nitrite ${ }^{8}$ and these have become popular as initial screening tests in general practice. ${ }^{9}$ Previous studies of dipstick tests performed urine analysis on collected urine samples (clean catch, bag, or catheter specimens) and showed sensitivity and negative predictive values of up to $100 \%$ in some settings. ${ }^{10-12}$ However, these collection methods are not used often in primary care for febrile or non-specific illnesses in young children.

A possible, alternative method to urine collection uses an absorbent panty-liner placed 
Table 1 Comparison of stick leucocyte esterase tests and laboratory outcomes

\begin{tabular}{lllllc}
\hline & \multicolumn{2}{l}{ Bayer stick } & & \multicolumn{2}{l}{ Boehringer stick } \\
\cline { 2 - 3 } Microscopy confirmation of pyuria & Negative & Positive & & Negative & Positive \\
\hline No & 50 & 3 & & 46 & 7 \\
Yes & 16 & 9 & & 7 & 18
\end{tabular}

Negative, leucocyte esterase was not detected on Boehringer, and "trace" or not detected on Bayer.

Bayer tests: sensitivity $36 \%$, specificity $94.3 \%$, negative predictive value $75.8 \%$.

Boehringer tests: sensitivity $72 \%$, specificity $86.8 \%$, negative predictive value $86.8 \%$.

Table 2 Comparison of stick nitrite tests and laboratory outcomes

\begin{tabular}{llllll}
\hline & \multicolumn{2}{c}{ Bayer stick } & & \multicolumn{2}{c}{ Boehringer stick } \\
\cline { 2 - 3 } Laboratory confirmation of UTI & Negative & Positive & & Negative & Positive \\
\hline No & 60 & 1 & 53 & 8 \\
Yes & 8 & 9 & & 5 & 12
\end{tabular}

Positive, nitrite was detected.

Bayer tests: sensitivity $52.9 \%$, specificity $98.4 \%$, negative predictive value $88.2 \%$.

Boehringer tests: sensitivity $70.6 \%$, specificity $86.9 \%$, negative predictive value $91.4 \%$.

Table 3 Comparison of combined urine analysis with Bayer or Boehringer sticks performed on liquid urine sample and laboratory outcomes

\begin{tabular}{lllllll}
\hline & \multicolumn{3}{l}{ Bayer stick } & & \multicolumn{2}{l}{ Boehringer stick } \\
\cline { 2 - 3 } Laboratory confirmation of UTI & Negative & Positive & & Negative & Positive \\
\hline No & 58 & 3 & & 46 & 15 \\
Yes & 4 & 13 & & 1 & 16
\end{tabular}

Positive, either leucocyte esterase or nitrites or both were detected; Negative, neither were detected. ("Trace" leucocyte esterase on Bayer was classified as negative.)

Sensitivity, specificity, and negative predictive values were calculated from these results and compared with those from testing urine soaked onto panty-liners (table 4), together with negative predictive values were calculated assuming UTI prevalences of $1 \%$ or $5 \%$.

inside an infant's nappy; the absorbed urine is tested with urine analysis dipsticks. Small amounts of urine are absorbed too efficiently into new gel bead infant nappies, but pantyliners are less absorbent and thin enough to be placed inside the nappy without discomfort. When the infant produces urine, the liner becomes damp enough for urine analysis with dipsticks: the stick is simply pressed on to the wet area of the liner. We set out to examine the accuracy of this method by an initial in vitro experiment. We used samples collected from patients attending routine nephro-urology outpatient clinics, assessing the accuracy of urine analysis on panty-liners compared with laboratory investigation results.

\section{Methods}

Urine samples from 91 outpatients attending nephro-urology clinics were divided into three $5 \mathrm{ml}$ aliquots. The first was sent for routine laboratory analysis (microscopy and culture) after dipstick analysis with Bayer Multistix 8SG (Bayer Diagnostics, Bayer plc, Basingstoke, UK) and Boehringer Mannheim Nephur sticks (Boehringer Manngeim UK, (Diagnostics and Biochemicals) Ltd, Lewes, East Sussex, UK). The remaining $5 \mathrm{ml}$ urine aliquots were poured on to Sainsbury's and Boots' proprietary panty-liners and each was tested directly with both urine analysis sticks. All urine analysis was undertaken by one investigator (HT).

From each patient's sample, the results of leucocyte esterase and nitrite tests were recorded for the six combinations of urine aliquot (three samples) and urine analysis stick (two manufacturers), as were the results of laboratory investigation. Laboratory UTIs were defined as a pure growth of over 100000 bacteria per millilitre. ${ }^{5}$ We have assumed that samples with a contaminated growth on culture were truly contaminated and classified them among the normal/negative results.

\section{STATISTICAL ANALYSES}

Initial analyses were made for leucocyte esterase and nitrites separately, comparing with leucocytes on microscopy and laboratory identified UTIs, respectively (the local laboratory does not routinely record the presence of organisms on microscopy).

The clinical value of urine analysis is more likely to depend on a combination of the leucocyte esterase and nitrite readings, in particular the accuracy of urine analysis, which is negative for both elements. Therefore, we also assessed the sensitivity, specificity, and negative predictive value of this "negative urine analysis" in relation to laboratory identified UTIs. As negative predictive value is prevalence dependent, and the prevalence in this setting is likely to be higher than would be encountered in primary care, negative predictive values were also calculated assuming prevalences of UTI of $1 \%$ or $5 \%$, with $95 \%$ confidence intervals (CI) based on the ratio of two proportions. ${ }^{13}$ Confidence intervals for $\kappa=1$ were imputed as the corresponding $\mathrm{CI}$ for the difference between two proportions ${ }^{14}$ and $95 \%$ CI for bias were calculated appropriately (Newcombe RG, 1997, unpublished data).

\section{Results}

Urine samples were obtained from 91 patients (mean age 41.3 years; range 3 months to 83 years). Laboratory results were available for 78 patients, of which $17(21.8 \%)$ had UTIs. The comparisons of conventional leucocyte esterase and nitrite tests with laboratory outcomes are shown in tables 1-3. From this baseline, differences in sensitivity, specificity, and negative predictive value for combined urine analysis tests by different methods (conventional urine analysis or panty-liners) were examined for bias and agreement. These results are shown in table 4, which also includes projections for a lower prevalence setting, as would be expected in general practice.

From cross-tabulations of test results, we assessed the disagreement proportions between pairs of methods to indicate bias. The point estimate of differences between methods ranged from 0-0.06 for all pairs comparing urine testing with panty-liner testing for either leucocyte esterase or nitrite with a given dipstick. The $95 \%$ CI included zero in all cases, indicating no evidence of bias between methods for urine analysis, although the upper limit was 0.15 , which is potentially important but reflects the limitations of the small sample size.

As nitrite test results are either positive or negative, Cohen's $\kappa$ statistic was calculated to assess the degree of agreement between methods, over and above that which would be expected by chance, comparing for example 
Table 4 Comparisons of accuracy of methods to identify laboratory confirmed urinary tract infections

\begin{tabular}{|c|c|c|c|c|c|}
\hline Analysis & Sensitivity \% & Specificity \% & $N P V \%$ & $\begin{array}{l}\text { NPV at } 5 \% \\
\text { prevalence }\end{array}$ & $\begin{array}{l}\text { NPV at } 1 \% \\
\text { prevalence }\end{array}$ \\
\hline Direct urinalysis, Bayer sticks & 76.5 & 95.1 & 93.5 & & \\
\hline Result if LE trace was considered positive ${ }^{\star}$ & 76.5 & 91.8 & 93.3 & 98.7 & 99.8 \\
\hline Sainsbury liner, Bayer sticks & 76.5 & 94.8 & 93.2 & & \\
\hline Result if LE trace was considered positive ${ }^{\star}$ & 76.5 & 91.4 & 93.0 & 98.7 & 99.7 \\
\hline Boots liner, Bayer sticks & 75.0 & 98.1 & 92.9 & & \\
\hline Result if LE trace was considered positive ${ }^{\star}$ & 81.3 & 88.7 & 94.0 & 98.7 & 99.7 \\
\hline Direct urinalysis, Boehringer sticks & 94.1 & 75.4 & 97.9 & 99.6 & 99.9 \\
\hline Sainsbury liner, Boehringer sticks & 94.1 & 69.0 & 97.6 & 99.6 & 99.9 \\
\hline Boots liner, Boehringer sticks & 93.7 & 69.8 & 97.4 & 99.5 & 99.9 \\
\hline
\end{tabular}

* Same experimental results reanalysed classifying trace leucocyte esterase (LE) as positive.

$95 \%$ confidence intervals (CI) for the Bayer NPVs (all methods) at $5 \%$ prevalence were $97.4-99.5 \%$; at $1 \%$ prevalence, $95 \%$ CI (all methods) were $99.5-99.9 \%$.

95\% CI for the Boehringer NPVs at 5\% prevalence were $97.9-99.9 \%$; at $1 \%$ prevalence, $95 \%$ CI (all methods) were $99.6-100 \%$.

Bayer tests, direct urine versus Sainsbury's liner. For Bayer tests, complete agreement was observed between direct urine and both liners. (Boots and direct urine analysis: $\kappa,+1 ; 95 \%$ CI, 0.69-1.0; Sainsbury and direct urine analysis: $\kappa,+1 ; 95 \%$ CI, 0.74-1.0.) For Boehringer, there was agreement in over $85 \%$ of cases. (Boots and direct urine analysis: $\kappa$, $0.61 ; 95 \%$ CI, 0.41-0.82; Sainsbury and direct urine analysis: $\kappa, 0.66 ; 95 \% \mathrm{CI}, 0.48-0.84$.)

\section{Discussion}

Urine analysis on panty-liners soaked with urine is feasible and achieves a consistent result when compared to the usual analysis of liquid urine samples for leucocyte esterase and nitrites. This study has used urine from a mostly adult sample, but shows that the method offers a potential solution to the problem of investigating children in primary care with non-specific or febrile illnesses and, thus, could make it easier for general practitioners to consider a diagnosis of UTI. Dipstick urine analysis is already popular and established in primary care, ${ }^{9}$ but urine collection from non-toilet trained infants is difficult and time consuming in practice. ${ }^{7}$

A preliminary and rapid urine analysis test on the child's urine would be possible and initial clinical decisions could be made without waiting for laboratory investigations. A negative result would confidently exclude UTI, and a positive result would identify those in whom microbiological urine culture was necessary to confirm or refute a diagnosis of UTI. If widely implemented in practice, the advantages of this method would include reduced time spent in the surgery by patients awaiting investigations, and increased ascertainment of childhood UTI among those identified as requiring microbiological urine culture. Improved management would be likely to reduce the incidence of renal scarring. ${ }^{6}$

Agreement between individual stick test results for leucocyte esterase or nitrite and the laboratory results is poor, but combining the analysis for both leucocyte esterase and nitrite improves accuracy considerably, as in other studies. ${ }^{11}$ Specificity and sensitivity of combined urine analysis varied according to the stick type used, and was not as high as reported elsewhere. ${ }^{10}{ }^{11}$ In this analysis, with "trace" leucocyte esterase classified as negative on Bayer sticks, Bayer results had a higher specificity and a lower sensitivity than Boehringer. This remains so if trace is included as positive, although the difference is less marked. Which is most useful clinically will vary with the clinical situation. $^{15}$

The sensitivities of $76-94 \%$ make urine analysis unsuitable for preliminary investigation in all clinical situations, whether on liners inside nappies or collected samples. If the prior probability of UTI is high, such as in children with recurrent infections or immunosuppression, accurate exclusion of UTI in these young children still requires laboratory investigation of collected urine. ${ }^{16}$

However, urine analysis is likely to be useful in primary care. A low false negative rate is crucial, as failure to identify and treat a true UTI causes persistence of current morbidity, and risks future renal scarring, hypertension, etc. ${ }^{2}$ Compromising specificity is inevitable, but worthwhile, to achieve higher sensitivity: the only adverse consequence of a false positive urine analysis result is the need for further laboratory investigation. The yield of infected urine will, however, be higher than at present as the majority of infection free samples are excluded.

The clinical usefulness of urine analysis depends not only on high sensitivity but also on a high negative predictive value. In this study population, the prevalence of UTI was $21.8 \%$, and yet urine analysis still had a negative predictive value of $93.5 \%$ (Bayer) or $97.9 \%$ (Boehringer). Urinary tract infections are more likely to have a prevalence of $1-5 \%$ in the general practice setting. ${ }^{1}$ The projections for negative predictive value at $1 \%$ and $5 \%$ prevalences show that with these sensitivities and specificities, UTI could be excluded with confidence in most cases (over 99\%). The minority in whom efforts should be made to collect urine for investigation would also be identified.

For full implementation of this method in practice two areas of study are required: practical trials of the methodology on young children in nappies and the methodology and analysis in this study should be repeated on more urine samples from children. We need to know whether the assessment of urine on liners inside nappies can overcome the difficulties of contamination, and whether the time taken for the whole procedure will make it feasible in clinical practice. It will be attractive to general practitioners if it is both accurate and time sav- 
ing. Kits could be developed to enable parents to perform the whole test themselves. ${ }^{16}$ In the majority of children, a second urine void or collection would be unnecessary and, hence, it may be a more efficient and expedient process overall than, for instance, asking urine to be collected from all children reporting relevant symptoms.

More importantly, however, the method does require validation in very young children to examine its reliability and negative predictive value. Potentially, the results might not be as accurate for three reasons: (a) contamination from soiling or skin flora may impair accuracy; (b) children in this age group may not mount as strong a pyuric response as older subjects, thus influencing testing for leucocyte esterase; and (c) the nitrite test might not maintain such a high negative predictive value when testing children's urine, as the shorter duration of urinary stasis in the bladder prior to micturition limits production of detectable levels of nitrite. Leucocyte esterase and nitrite tests have, however, been shown elsewhere to produce accurate results in young children so they would not be expected to produce dramatically different results. ${ }^{11} 12$

If these issues are clarified, the testing of panty-liner samples of urine could be as accurate as testing actual urine samples. This would provide a potential method of analysis of suspected UTIs using leucocyte esterase and nitrite sticks on nappies. This would be a simpler, cheaper, preliminary test than microbiological investigation for young children presenting in primary care with febrile or nonspecific illnesses. In current practice, urine testing is an infrequent occurrence relative to the presentation of sick children in primary care. ${ }^{7}$ Wider implementation of urine testing, even with the limitations of sensitivity and specificity identified, would be a step forward in the management of UTI and prevention of complications such as renal scarring.

\section{CONCLUSIONS}

Urine analysis has been shown to produce consistent results when testing urine directly or on panty-liners. This method offers a potential solution to the problems of investigating UTI in non-toilet trained children in primary care who present with non-specific or febrile illnesses. Both brands of urine analysis stick have a high negative predictive value which would make them useful in a low prevalence setting such as general practice. These findings should facilitate ongoing efforts to raise professional and public awareness of the importance of UTI and encouraging clinicians to consider the diagnosis and undertake appropriate investigations.

The authors thank Roberta Roberts for her contribution to this work in processing the results for analysis, Stephen Granier for contributing to the analysis, and $\operatorname{Dr} \mathrm{R}$ Barnes, consultant microbiologist at the University Hospital of Wales. We also acknowledge the Kidney Research Unit Foundation for Wales and the Laura Ashley Foundation for general support to the Child Health Department at Cardiff Royal Infirmary. Funding for equipment was provided by Boehringer Mannheim and Bayer Pharmaceuticals.

1 Hoberman A, Chao H, Keller DM, Hickey R, Davies HW, Ellis D. Prevalence of urinary, Ellis D. Prevalence of urinary trac

2 Jakobsson B, Berg U, Svensson L. Renal scarring after acute pyelonephritis. Arch Dis Child 1994;70:111-15.

3 Winberg J, Bergstrom T, Jacobsson B. Morbidity, age and sex distribution, recurrences and renal scarring in symptomatic urinary tract infection in childhood. Kidney Int 1997; 8S: $101-6$.

4 Berg UB, Johansson SB. Age as a main determinant of renal functional damage in urinary tract infection. Arch Dis Child 1983;58:963-9.

5 Report of a Working Group of the Research Unit RC. Guidelines for the management of acute urinary tract infection in childhood. $\mathcal{F}$ Roy Coll Phys Lond 1991;25:36infecti

6 Winter AL, Hardy BE, Alton DJ, Arbus GS, Churchill BM. Acquired renal scars in children. $\mathcal{F}$ Urol 1983;129:1190-4. 7 van der Voort J, Edwards A, Roberts R, Verrier Jones K. The struggle to diagnose urinary tract infection in children under two in primary care. Fam Pract 1997;14:44-8.

8 Voswinckel P. A marvel of colors and ingredients. The story of urine test strips. Kidney Int 1994;46(Suppl 47):S3-7.

9 Ditchburn RK, Ditchburn JS. A study of microscopical and chemical tests for the rapid diagnosis of urinary tract infecchemical tests for the rapid diagnosis of urinary tract inf

10 Woodward MN, Griffith DM. Use of dipsticks for routine analysis of urine from children with acute abdominal pain. $B M F$ 1993;306:1512.

11 Hiraoka M, Hida Y, Tuchida S, Kuroda M, Sudo M. Rapid dipstick test for diagnosis of urinary tract infection. Acta Paediatr fap 1994;36:379-82.

12 Lejeune B, Baron R, Guillois B, Mayeux D. Evaluation of a screening test for detecting urinary tract infection in newborns and infants. $\mathcal{F}$ Clin Pathol 1991;44:1029-30.

13 Miettinen OS, Nurminen M. Comparative analysis of two rates. Stat Med 1985;4:213-26.

14 Newcombe RG. Interval estimation for the difference between independent proportions. A comparative evaluation of eleven methods. Stat Med 1997. [In press.]

15 Edwards A, Granier S, van der Voort J. Usefulness of urine dipstick tests: packaging may lead to false positive results [letter]. BMf 1996;313:1010

16 Hashmi P, Ho C, Morgan S, Stephenson JR. Routine analysis in renal transplant patients. $\mathcal{f}$ Clin Pathol 1995;48:383-4. 